\title{
Direct Writing and Electro-Mechanical Characterization of Ag Micro-Patterns on Polymer
}

\section{Substrates for Flexible Electronics}

\author{
Maria A. Torres Arango ${ }^{1}$, Anna M. Cokeley ${ }^{1}$, Jared J. Beard ${ }^{1}$, Konstantinos A. Sierros ${ }^{\mathbf{1}^{*}}$ \\ ${ }^{1}$ West Virginia University, Department of Mechanical \& Aerospace Engineering, 395 Evansdale \\ Drive,Morgantown, WV, 26505, USA
}

\begin{abstract}
There is currently a great interest in developing flexible electrodes. Such components are used in most electronic devices from displays to solar cells to flexible sensors. To date most of them are fabricated using expensive vacuum techniques, and are based on transparent conducting oxides. These oxides are not entirely compatible with flexible substrates under the application of mechanical stresses, due to their brittle nature. Therefore, there is a need to explore novel lowcost, large-area fabrication methods to deposit alternative conducting materials with enhanced electro-mechanical performance.
\end{abstract}

This work focuses on $\mathrm{Ag}$ patterns fabricated at low temperatures (below $150^{\circ} \mathrm{C}$ ) on flexible polyethylene naphthalate utilizing a robotic printing approach. Such lithography-free method minimizes material waste by printing exact amounts of inks on digitally predefined locations. Additionally, it allows a broad feature size range, from a few $\mathrm{m}$ to a few $\mathrm{mm}$, and a variety of ink viscosities for better pattern control.

We investigate the synthesis and direct writing of Ag particle-based inks, patterned-on-flex as lines and grids in the m scale. We report on a high-yield ink synthesis method $(\sim 61.6 \%)$ with controlled particle size. It is found that the electrical resistivity $\left(1.75^{*} 10^{-4} \Omega \mathrm{cm}\right)$ of the patterns is in the same range with similar particle-based conductive components. The correlation between annealing temperature, microstructural evolution, and electrical performance is established. Also, 
the optical transmittance of the patterns can be controlled to meet specific application requirements by regulating the substrate surface area covered. Finally, the mechanical behavior under both monotonic and cyclic conditions shows a superior performance compared to brittle counterparts and underlines the potential of such metallic micro-patterns to be utilized in a wide range of flexible electronic applications. It is believed that direct writing of Ag patterns on compliant substrates may hold the key in developing the next generation of truly flexible devices.

Keywords: Direct writing, Ag patterns, flexible electronics, compliant substrates.

*E-mail:knostas.sierros@mail.wvu.edu , phone: +1 304293 3420, fax: +1 3042936689

\section{Introduction}


There is currently a great interest to advance the development of large-area transparent conducting electrodes (TCEs). TCE components are integrated into a range of flexible optoelectronic applications spanning from displays to touch sensors to solar cells [1]. Transparent conducting oxides (TCOs), such as indium tin oxide (ITO), are the current solution for such applications due to their excellent electrical and optical properties [2]. In particular, ITO exhibits the lowest resistivity in the TCO group in the order of1-2*10 ${ }^{-4} \Omega \mathrm{cm}$ and optical transmittance, at $550 \mathrm{~nm}$ wavelength, above $92 \%$ [3].

However, due to the scarcity of indium, the need for vacuum processing, and the limited mechanical flexibility of TCOs, owing to their brittle nature [4-8], there is a need to develop alternative TCEs [9] on mechanically flexible, low temperature, polymeric substrates. Currently explored alternatives include carbon nanotube films [10], found to exhibit enhanced mechanical flexibility compared to ITO but requiring electrical conductivity improvements [11]. In addition, the candidates to replace TCOs encompass electrically conductive polymers, such as poly-(3, 4ethylenedioxythiopene): poly (styrenesulfonic acid) (PEDOT:PSS), which exhibit reversible stretchability up to $10 \%$ uniaxial tensile strain when pre-strained on silicone substrates [12].Nevertheless, the electrical stability of PEDOT: PSS needs to be further improved since its conductivity and environmental stability are observed to decrease upon exposure to non-inert environments [13]. Some recent efforts focus on fabricating highly flexible, optically transparent graphene TCEs and graphene-conducting polymer hybrids $[14,15]$ but the need to further develop and refine large-area and defect-free graphene synthesis methods remains a challenge before the widespread adoption of such electrodes[16]. Additionally, the use of metallic nanostructures such as $\mathrm{Ag}$ nanowires is another alternative that is currently being investigated with the challenge to improve its atmospheric corrosion resistance [17]. 
Capitalizing on the high electrical conductivity of Ag, and other metals, is of paramount importance in flexible electronics since the utilization of Ag grids [18] is another path for flexible electrode development [19] and, even beyond TCEs, towards other applications such as flexible antennas for RFID applications [20] and strain gauge sensors [21]. However, most of the methods for fabricating such grid nano/micro-patterns are of relatively high cost or involve multi-step lithographic methods which do not offer a large-area processing potential [22]. In order to overcome such challenges novel and low-cost direct patterning methods are needed to provide large-area substrate coverage. Ink-jet printing of Ag nanoparticle-based patterns has been utilized [23] but nozzle clogging remains an issue to be resolved. Screen printing of thick Ag paste is another currently employed direct patterning method [24] with comparatively large material waste during the process. More recent efforts focus on filamentary printing approaches using relatively high viscosity Ag inks where the concentrated ink solution is extruded through a cylindrical nozzle using a robotic $\mathrm{X}-\mathrm{Y}-\mathrm{Z}$ stage. This direct writing approach offers significant advantages compared to ink-jet printing, such as large-area and precise ink placement even on curved surfaces, less clogging-related issues, and ability to write in three-dimensions (3D)[25].Quite recently, well-defined Ag nanoparticle-based periodic arrays, printed on glass and polyimide, with center-to-center separation distances of $400 \mu \mathrm{m}$ and using an annealing temperatures above $200{ }^{\circ} \mathrm{C}$ were demonstrated [26].However, the ink yield from this processing route has not been emphasized [27].

In this work we report on the synthesis of high-yield Ag inks and their robotic-based direct micro-patterning on polyethylene naphthalate $(\mathrm{PEN})$ substrates. We focus on relatively low Ag annealing temperatures (up to $150{ }^{\circ} \mathrm{C}$ ) suitable for heat-sensitive polymer substrates and we investigate the resulting electrical and mechanical cyclic loading behavior. 


\section{Materials and experimental methods}

\section{1: Ink synthesis and characterization}

The Ag ink is obtained through preparation of Ag nanoparticles through a modification of the solution-based process described in the work conducted by Ahn et al. [25]. Briefly, Ag particles are prepared from silver nitrate $(10.19 \mathrm{~g})$, poly acrylic acid (PAA) $(0.57 \mathrm{~g})$ and ethanolamine $(8.90 \mathrm{~g})$ mixed in water $(35.9 \mathrm{~g})$. Our approach consists of the following steps: first, the (PAA) was dissolved in half the water while magnetically stirring and the ethanolamine was then added dropwise to the stirring water-PAA solution. In parallel, the silver nitrate was dissolved in the remaining water and dropwise incorporated. The solution was stirred at $60^{\circ} \mathrm{C}$ for $\sim 14$ hours; and it was further stirred without heat for 2 hours to bring it to room temperature. The resulting colloidal dispersion was brown-black in color. This suspension was centrifuged at $4400 \mathrm{rpm}$ for 30 minutes to collect the PAA/Ag particles. The collected precipitates were re-dispersed using ultrasonication to form the ink (70 wt.\% solids, $29.4 \mathrm{wt} . \%$ water and $0.6 \mathrm{wt} \%$ methyl cellulose). After 15 days, the remaining Ag concentrated fluid was centrifuged and Ag/PAA precipitates were collected. Again, re-dispersion of the solids to form the ink was done. The particle size of the Ag/PAA precipitates was measured by dynamic light scattering with a Zetasizer Nano-ZS ZEN 3600, Malvern Instruments. The viscosity of the ink was measured with a Brookfield DVII+ Pro rotational viscometer at various shear rates.

\section{2: Pattern printing and annealing}

The electrically conducting patterns were directly written using a nozzle based robotic deposition (NBRD) method utilizing a Nordson JR2300N robotic arm equipped with a Performus V pneumatic pressure ink dispenser system. The base PEN substrates consist of biaxially oriented - 
TeonexQ65 films from DuPont Teijin Films, UK; and were used as received since they come with a protective film that can be manually removed just before processing. Therefore, the actual surface for printing was only exposed to ambient conditions a few seconds ( $\sim 1-2 \mathrm{~s})$ prior to printing to avoid contamination. For the deposition, a $100 \mu \mathrm{m}$ diameter stainless steel nozzle was used. The writing speed as well as the deposition pressure were varied to evaluate their effect on the printing process. The resulting patterns consisted of parallel lines drawn with a center to center spacing of $0.5 \mathrm{~mm}$ and $1 \mathrm{~mm} ; 90^{\circ}$ grids and $45^{\circ}$ grids. The printed patterns were then annealed in a box furnace KSL $1100 \mathrm{X}$, MIT Corporation; at $90^{\circ} \mathrm{C}, 120^{\circ} \mathrm{C}$ and $150^{\circ} \mathrm{C}$ for 30 minutes with a heating rate of $5^{\circ} \mathrm{C} \mathrm{min}^{-1}$.

\section{3: Pattern characterization}

Contact angle data were obtained through image analysis of droplets of both distilled water and formulated Ag ink on PEN using ImageJ software (NIH). Optical images of the printed patterns were taken with a Dino Edge-Digital programmable optical microscope at magnifications of 20x and 175x. Scanning electron microscope (SEM) images were obtained using a Hitachi S-4700 SEM machine at $5 \mathrm{kV}$ accelerating voltage and $12 \mathrm{~mm}$ working distance; imaging of the patterns was performed without any sample preparation or coating since the Ag patterns are electrically conductive. Particle size analysis of the Ag surfaces was conducted, from the obtained SEM images, by image analysis software (ImageJ). The size of the particles and their statistical distribution were calculated using the particle analysis feature. To do so, the images are converted to binary-color scheme images, then an appropriate range of possible size and shape (diameter and circularity) is given, based on the SEM raw data, for the analyzer to fit the background enclosed areas (particles) within a given area (total image). Then, the software can identify the number of individual contrasting areas (particles) and their size. X-ray diffraction spectra were obtained with a Bruker D8 Discovery XRD machine in 1D mode and 40kV - 
40mAmp configuration. Electrical resistivity measurements were taken by Hall Effect using an Ecopia HMS-3000 measurement system with a magnetic field of 1.02T. Light transmittance measurements were taken between 500 - $900 \mathrm{~nm}$ of wavelength with a JAZ UV-Vis Spectrometer Ocean Optics. Finally, for the mechanical characterization, cyclic loading testing was performed using an ADMET MTESTQuattro mechanical testing unit, employing a tensile sawtooth profile with a servo control displacement rate of $2 \mathrm{~mm} / \mathrm{min}$ up to $2 \%$ strain. Electrical resistance variation was monitored in-situ using a digital multimeter Agilent 34970Aalong with a Dino edge-Digital optical microscope to monitor the sample surface.

\section{Results and discussion}

During the preparation of the Ag nanoparticles ethanolamine was used instead of diethanolamine as the weak base precursor for the reduction process of silver ions for particle nucleation [25, 28]. The lower viscosity of ethanolamine allows for relatively easy extraction of the nanoparticles from the precursor solution. Also, the stronger $\mathrm{pH}$ of ethanolamine allows for utilization of less amounts of precursor to induce particle formation and growth.

The Ag yield was calculated from the approximate Ag content in the precipitates from the PAA/Ag precursor ratio and it was found to be $\sim 11.2 \%$ right after preparation of the particles; and $\sim 61.6 \%$ after 15 days. At this stage, it is important to note that the particle size, as measured from DLS for both points in time, was found to increase only by $7.41 \%$ which confirms the stabilizing role that PAA plays as the capping agent in the system. The observed improvement in yield is of paramount importance in processing because it suggests routes for yield control without the associated particle coarsening, towards higher processing efficiencies. Also, the fact that the particles do not exhibit significant growth in size when immersed in the precursor 
solution, suggests that the growth mechanism is strongly controlled by the thermal energy addition at the initial nucleation-growth stage; i.e. the growth of such particles occurs mainly while the precursor solution is heated at $60^{\circ} \mathrm{C}$ in the initial stirring process, rather than while the solution ages at room temperature for $\sim 15$ days.

The obtained ink allows for printing 2-dimensional (2D) electrically conductive Ag micropatterns. It exhibits shear thinning behavior as shown in Figure 1. Tailoring of the viscosity can be performed by varying the particle loading and the polymer content. Such tailoring would allow for writing of finer resolution features or even fabricating 3D structures. The measured viscosity of the synthesized ink is observed to lay in the range expected for $2 \mathrm{D}$ array printing (0.05-0.1 $\mathrm{Pa} \mathrm{s})$ and is comparable to similar systems reported in the past[25].

In order to investigate the printing process reliability, a range of extrusion pressures and writing speeds were utilized. In general, speeds between 3 and $10 \mathrm{~mm} / \mathrm{s}$ and pressures of 68.9 to $137.9 \mathrm{kPa}$ lead to patterns of width in the $\mu \mathrm{m}$ range. Figure 2 depicts different working combinations of these two parameters. It is observed that $7 \mathrm{~mm} / \mathrm{s}$ and $137.9 \mathrm{kPa}$ (Figure 2c) result in well-defined patterns. In this particular case the spreading is around 2 times the inner nozzle diameter and occurs due to the viscous properties of the ink. Spreading may be desirable for providing larger area of contact between the ink and substrate. Spreading can be controlled, as shown in Figure 2a, by using lower pressures at the expense of pattern continuity, or by increasing the writing speed as shown in Figures $2 \mathrm{~b}$ and $2 \mathrm{c}$.

Furthermore, the electrical resistivity of the conducting patterns as a function of annealing temperature can be observed in Figure 3. Lower resistivity, and therefore higher electrical conductivity, is obtained for the patterns as temperature increases. The values for resistivity are comparable to those obtained for similar particle based Ag systems [26] annealed at higher 
temperatures. They are also comparable to the highest electrical resistivity reported for annealed ITO on glass [3]. However, the values for $150^{\circ} \mathrm{C}$ are still two orders of magnitude above those for bulk silver, which is in the order of $10^{-6} \Omega \mathrm{cm}$, indicating the potential for even better performance of Ag patterns on heat sensitive plastic substrates. Particularly, understanding of the particle surface binding phenomena and the effect of the polymeric compounds present in the formulated ink, may be the key towards achieving higher electrical conductivity [29].

The microstructure of the Ag pattern surfaces annealed at different temperatures can be observed in Figure 4. Spherical particles of $\sim 300 \mathrm{~nm}$ can be identified for all annealing conditions. Image analysis indicates that the surface particle size exhibits a slight increase as temperature increases. The latter is in agreement with the decreasing electrical resistivity tendency observed previously for increasing annealing temperature. The particle size results from the SEM image analysis are summarized in Table 1. XRD spectra, shown in Figure 5a, evidence the crystalline nature of the Ag patterned features, in accordance with PDF 00-004-0783. Broad peaks show around $2 \theta$ values of $49^{\circ}$ and $55.5^{\circ}$ corresponding to the PEN substrate. Figure $5 b$ shows the (111) reflection for different annealing conditions. It is observed that there is peak narrowing (FWHM) as temperature increases, indicating grain size increase. Particularly, using Scherrer's formula [30] we can approximate such increase in size which ranges from $\sim 46.93 \mathrm{~nm}$ for $90^{\circ} \mathrm{C}$ to $\sim 50.98 \mathrm{~nm}$ for $150^{\circ} \mathrm{C}$. Accordingly, contact area between particles is expected to increase. Such grain sizes are smaller than the particles size as measured from SEM images. In addition, reflection peak slight shifting is noted indicating presence of residual stress in the directly written film.

Figure 6 shows the different pattern configurations, and their respective UV-Vis spectra. Higher light transmission is measured for patterns with smaller percentages of Ag covered area (see Table 2); as is the case of parallel lines with variable center-to-center spacing of $500 \mu \mathrm{m}$ and $1000 \mu \mathrm{m}$, respectively. For the case of the grids, a fixed spacing of $500 \mu \mathrm{m}$ is considered. 
However, they cover a larger area on the substrate and their optical transmittance is compromised. The oscillation of the UV-Vis curves correspond to Fabry-Pérot fringes that result from the interference of partially transmitted and partially reflected light at the Ag surface/air and $\mathrm{Ag} / \mathrm{PEN}$ interfaces $[31,32]$

The mechanical behavior of such structures is of paramount importance for flexible electronics manufacturing and service conditions. The monotonic uniaxial tensile loading of Ag micropatterned lines -Figure 7- shows that the Ag film does not fail up to $10 \%$ applied strain. At this strain, using in-situ optical microscopy we observe that the underlying acrylic-based layer (2-4 nm thick - coated on PEN surface to promote adhesion[33]) fails by forming cracks that are perpendicular to the applied stress direction. The latter can be due to the UV-induced brittleness of the cured planarising layer.[33] Since the Ag patterns remain intact at relatively high strains, significantly above those that TCOs can sustain [4], the next step is to investigate their performance at cyclic loading conditions. We use $2 \%$ strain, which is the failure threshold for TCO's on polymeric substrates $[5,7]$ and we arbitrarily choose $20 \%$ increase in electrical resistance as the onset for failure. In Figure 8a, the normalized change in electrical resistance is shown as a function of the number of tensile loading - unloading cycles. The inset showing the first twenty cycles clearly depicts electrical recovery behavior upon release of the tensile load; i.e. the electrical resistance increases as load is applied and similarly, it decreases as the load is withdrawn. The recovering behavior is likely to be attributed to the organic-inorganic nature of the ink and its low temperature annealing which are both being restricting factors to particle coalescence and necking [34]. Therefore, it is believed that once the tensile stress is applied, the interparticle distance increases along the applied stress direction, resulting in a slight increase of resistance due to the fewer conducting paths available. Then, as the stress is released, the distance between particles within the film is restored along with the available conducting paths, leading to 
electrical resistance recovery. This result may suggest that the printed patterns can be utilized in applications for strain sensors. Furthermore, from the optical images in Figure 8b, no evidence of structural damage of the Ag film or the PEN substrate can be identified. To further investigate the tested surfaces, SEM images of the initial (i.e. before testing) and after 1000 cycles of loading were taken and are shown in Figure 9a and 9b, respectively. No apparent change or evidence of cracking or delamination at the surface of the Ag patterns is observed. We postulate, that as the samples are loaded, both the substrate and the Ag film deform simultaneously, and since Ag is ductile it is capable to withstand the load well within its elastic region [35]. The cumulative increase in electrical resistance is under the failure limit and can be attributed to slight neighboring particle relative displacements where the polymeric material may act as a binding and damping medium. In particular, since no delamination is observed it can be suggested that the stress transferred from the substrate to the film is not arrested at the film/substrate interface. It can also be suggested that the transferred stress is not causing multiplication of dislocations in the film for the applied strain range, as discussed by Kim et al. [34]; these researchers showed that film porosity allows for dislocation annihilation which, in turn, leads to reliable performance under cyclic applied stress conditions. At this stage we must note that the porous film microstructure observed in our work is similar to the aforementioned study for the respective annealing conditions.

\section{Conclusions}

The modified synthesis of the Ag ink allows for a higher yield ( $61.6 \%)$ without significant particle coarsening over time highlighting potential routes for enhancing processing efficiency. In particular, the incorporation of ethanolamine in the particle synthesis process allows for reduction 
of the amount of precursor to induce particle nucleation and growth due to its stronger $\mathrm{pH}$ when compared to its dimer.

The efficient direct NBRD micro-patterning of $\mathrm{Ag}$ on PEN at low temperatures (up to $150{ }^{\circ} \mathrm{C}$ ) is demonstrated. The resulting patterns exhibit electrical resistivity values comparable to other nanoparticle-based conductive systems. The electrical resistivity behavior is supported by the microstructural evolution with increasing annealing temperatures. The optical transmittance is correlated to substrate surface area covered by the pattern and therefore can be controlled to meet different requirements for specific applications. The mechanical performance during both monotonic and cyclic loading conditions highlights the potential of ductile Ag patterns as an alternative to brittle conductive components in applications even beyond TCEs.

\section{Acknowledgments}

K.A.S and M. A. T. A. greatly acknowledge the partial support of NSF under award no. 1343726. A. M. C. wishes to thank WVU's SURE NanoSAFE program for undergraduate research. J. J. B. acknowledges the support of WV NASA Space Grant Consortium for undergraduate research. We acknowledge use of the WVU Shared Research Facilities.

\section{References}

1. K. Ellmer, Past achievements and future challenges in the development of optically transparent electrodes, Nat. Photonics 6 (2012) 809-817.

2. D. S. Hecht, L. Hu, G. Irvin, Emerging transparent electrodes based on thin films of carbon nanotubes, grapheme and metallic nanostructures, Adv. Mater. 23 (2011) 1482-1513. 
3. U. Betz, M. Kharrazi Olsson, J. Marthy, M. F. Escola, F. Atamny, Thin films engineering of indium thin oxide: Large area flat panel displays application, Surf. Coat. Techn. 200 (2006) 5751-5759.

4. D. R. Cairns, R. P. Witte II, D. K. Sparacin, S. M. Sachsman, D. C. Paine, G. P. Crawford, R. R. R. Newton, Strain-dependent electrical resistance of tin-doped indium oxide on polymer substrates, Appl. Phys. Lett. 76 (2000) 1425-1427.

5. Y. Leterrier, L. Medico, F. Demarco, J. A. E. Manson, U. Betz, M. F. Escola, M. Kharrazi Olsson, F. Atamny, Mechanical integrity of transparent conductive oxide films for flexible polymer-based displays, Thin Solid Films 460 (2004) 156-166.

6. E. Fortunato, P. Nunes, A. Marques, D. Costa, H. Aguas, I. Ferreira, M. E. V. Costa, M. H. Godinho, P. L. Almeida, J. P. Borges, R. Martins, Transparent, conductive ZnO:Al thin film deposited on polymer substrates by RF magnetron sputtering, Surf. Coat. Techn. 151-152 (2002) 247-251.

7. K. A. Sierros, D. A. Banerjee, N. J. Morris, D. R. Cairns, I. Kortidis, G. Kiriakidis, Mechanical properties of $\mathrm{ZnO}$ thin films deposited on polyester substrates used in flexible device applications, Thin Solid Films 519 (2010) 325-330.

8. A. J. Stapleton, S. Yambem, A. H. Johns, C. T. Gibson, C. J. Shearer, A. V. Ellis, J. G. Shapter, G. G. Andersson, J. S. Quinton, P. L. Burn, P. Meredith, D. A. Lewis, Pathway to high throughput, low cost indium-free transparent electrodes, J. Mater. Chem. A 3(2015) 13892-13899.

9. A. Kumar, C. Zhou, The race to replace tin-doped indium oxide: which material will win?, ACS Nano 4 (2010) 11-14.

10. L. Hu, D. S. Hecht, G. Gruner, Percolation in transparent and conducting carbon nanotube networks, Nano Lett. 4 (2004) 2513 - 2517.

11. K. A. Sierros, D. S. Hecht, D. A. Banerjee, N. J. Morris, L. Hu, G. C. Irvin, R. S. Lee, D. R. Cairns, Durable transparent carbon nanotube films for flexible device components, Thin Solid Films 518 (2010) 6977-6983.

12. M. Vosgueritchian, D. J. Lipomi, Z. Bao, Highly conductive and transparent PEDOT:PSS films with a fluorosurfactant for stretchable and flexible transparent electrodes, Adv. Funct. Mater. 22 (2012) 421-428.

13. A. M. Nardes, M. Kemerink, M. M. de Kok, E. Vinken, K. Maturova, R. A. J. Janssen, Conductivity, work function, and environmental stability of PEDOT:PSS thin films treated with sorbitol, Org. Electron. 9 (2008) 727-734.

14. L. Gomez De Arco, Y. Zhang, C. W. Schlenker, K. Ryu, M. E. Thompson, C. Zhou, Continuous, highly flexible and transparent grapheme films by chemical vapor deposition for organic photovoltaics, ACS Nano 4 (2010) 2865-2873. 
15. K. Rana, J. Singh, J. H. Ahn, A grapheme-based transparent electrode for use in flexible optoelectronic devices, J. Mater. Chem. C 2 (2014) 2646-2656.

16. B. H. Lee, J. H. Lee, Y. H. Kahng, N. Kim, J. Lee, T. Lee, K. Lee, Graphene-conducting polymer hybrid transparent electrodes for efficient organic optoelectronic devices, Adv. Funct. Mater. 24 (2014) 1847-1856.

17. J. L. Elechiguerra, L. Larios-Lopez, C. Liu, D. Garcia - Gutierrez, A. Camacho - Bragado, M. J. Yacaman, Corrosion at the nanoscale: the case of silver nanowires and nanoparticles, Chem. Mater. 17 (2005) $6042-6052$.

18. R. B. Pode, C. J. Lee, D. G. Moon, J. I. Han, Transparent conducting metal electrode for top emission organic light-emitting devices: Ca-Ag double layer, Appl. Phys. Lett. 84 (2004) $4614-4616$.

19. Y. Li, L. Mao, Y. Gao, P. Zhang, C. Li, C. Ma, Y. Tu, Z. Cui, L. Chen, ITO-free photovoltaic cell utilizing a high-resolution silver grid current collecting layer, Sol. Energ. Mat. Sol. C. 113 (2013) 85-89.

20. A. Rida, L. Yang, R. Vyas, M. M. Tentzeris, Conductive inkjet-printed antennas on flexible low-cost paper-based substrates for RFID and WSN applications, IEEE Antenn. Propag. M. 51 (2009) 13-23.

21. A. Moorthi, B. B. Narakathu, A. S. G. Reddy, A. Eshkeiti, H. Bohra, M. Z. Atashbar, A novel flexible strain gauge sensor fabricated using screen printing, IEEE $6^{\text {th }}$ International Conference on Sensing Technology (ICST) (2012) 765-768.

22. M. G. Kang, L. J. Guo, Nanoimprinted semitransparent metal electrodes and their application in organic light-emitting diodes, Adv. Mater. 19 (2007) 1391-1396.

23. Z. Liu, Y. Su, K. Varahramyan, Inkjet-printed silver conductor using nitrate ink and their electrical contacts with conducting polymers, Thin Solid Films 478 (2005) 275-279.

24. F. C. Krebs, All solution roll-to-roll processed polymer solar cells free from indium-tin-oxide and vacuum coating steps, Org. Electron. 10 (2009) 761-768.

25. B. Y. Ahn, E. B. Duoss, M. J. Motala, X. Guo, S. I. Park, Y. Xiong, J. Yoon, R. G. Nuzzo, J. A. Rogers, J. A. Lewis, Omnidirectional printing of flexible, stretchable, and spanning silver microelectrodes, Science 323 (2009) 1590-1593.

26. B. Y. Ahn, D. J. Lorang, J. A. Lewis, Transparent conducting grids via direct writing of silver nanoparticle inks, Nanoscale 3 (2011) 2700-2702.

27. B. Y. Ahn, J. A. Lewis, Amphiphilic silver particles for conductive inks with controlled wetting behavior, Mat. Chem. Phys. 148 (2014) 686-691. 
28. B.H. Ryu, Y. Choi, H.S. Park, J.H. Byun, K. Kong, J.O. Lee, H. Chang, Synthesis of highly concentrated silver nanosol and its application to inkjet printing, Colloid Surface A 270 (2005) 345-351.

29. S. Magdassi, M. Grouchko, O. Berezin, A. Kamyshny, Triggering the sintering of silver nanoparticles at room temperature, ACS Nano 4 (2010) 1943-1948.

30. J. I. Langford, A. J. C. Wilson, Scherrer after sixty years: a survey and some new results in the determination of crystallite size, J. Appl. Cryst. 11 (1978) 102-113.

31. V.S.Y. Lin, K. Motesharei, K.P.S. Dancil, M.J. Sailor, M.R. Ghadiri, A porous silicon-based optical interferometric biosensor, Science 278 (1997) 840-843.

32. X. Liu, L. Zhou, F. Liu, M. Ji, W. Tang, M. Pang, J. Sun, Exponential growth of layer-bylayer assemble coating with well-dispersed ultrafine nanofiller: a facile route to scratchresistant and transparent hybrid coatings, J. Mater. Chem. 20 (2010) 7721-7727.

33. Adam R, Eveson RW, MacDonald WA, inventors; Dupont Teijin Films U.S. Limited Partnership, assignee. Coated and Planarised Polymeric Films US Patent 20110100454 A1, 2011 May 5.

34. B-J. Kim, T. Haas, A. Friederich, J-H. Lee, D-H. Nam, J. R. Binder, W. Bauer, I-S. Choi, YC. Joo, P. A. Gruber, O. Kraft, Improving mechanical fatigue resistance by optimizing the nanoporous structure of inkjet-printed Ag electrodes for flexible devices, Nanotechnology 25 (2014) 125706

35. D. R. Smith, F. R. Ficket, Low-temperature properties of silver, J. Res. Natl. Inst. Stand. Technol. 100 (1995) 119-171.

\section{Figure Captions}

Figure 1. Viscosity of the prepared Ag ink as a function of shear rate.

Figure 2. Optical images of directly written patterns exhibiting the effect of writing speed and deposition pressure variation.

Figure 3. Electrical resistivity measured by Hall Effect of the printed patterns for different annealing temperatures.

Figure 4. SEM images of the patterns surface (a) as printed $25^{\circ} \mathrm{C}$; and annealed at (b) $90^{\circ} \mathrm{C}$, (c) $120^{\circ} \mathrm{C}$ and (d) $150^{\circ} \mathrm{C}$. 
Figure 5. (a)XRD spectra of the printed Ag patterns annealed at different temperatures and the base substrate and (b) (111) reflection and respective Gaussian fits for the different annealing temperature conditions.

Figure 6. (a) Digital images of various printed Ag pattern configurations and (b) their respective optical transmission between 500-900 nm wavelengths.

Figure 7. Optical images of the Ag patterned surfaces at various uniaxial strains under monotonic loading conditions. Substrate failure by cracking can be observed for $10 \%$ strain.

Figure 8. (a) Normalized change in electrical resistance of Ag line patterns cyclically tested up to 2\% strain for 1000 cycles. Inset graph shows the first 20 cycles. (b) Optical images depicting the micro-patterned surface at different loading cycles.

Figure 9. Optical and SEM images of the microstructure of the line patterns sintered at $150^{\circ} \mathrm{C}$ (a) before cyclic loading and (b) after 1000 cycles of tensile loading up to $2 \%$ stain.

\section{Table Captions}

Table 1. Surface particle size dependence on annealing temperature. Calculated using image analysis.

Table 2. Area covered by Ag for different pattern configurations. Calculated using image analysis. 


\begin{tabular}{ccc}
\hline Temperature $\left({ }^{\circ} \mathrm{C}\right)$ & $\begin{array}{c}\text { Average Particle } \\
\text { Size }(\mathrm{nm})\end{array}$ & $\begin{array}{c}\text { Standard Deviation } \\
(\mathrm{nm})\end{array}$ \\
\hline $\mathbf{2 5}$ & 299 & 189 \\
$\mathbf{9 0}$ & 288 & 178 \\
$\mathbf{1 2 0}$ & 310 & 161 \\
$\mathbf{1 5 0}$ & 341 & 182 \\
\hline
\end{tabular}


Table 2

\begin{tabular}{cc}
\hline Pattern & Ag Area Covered (\%) \\
\hline Lines 2X spaced & 30.00 \\
Lines & 47.98 \\
Grid & 75.99 \\
$\mathbf{4 5}^{\circ}$ Grid & 76.02 \\
\hline
\end{tabular}




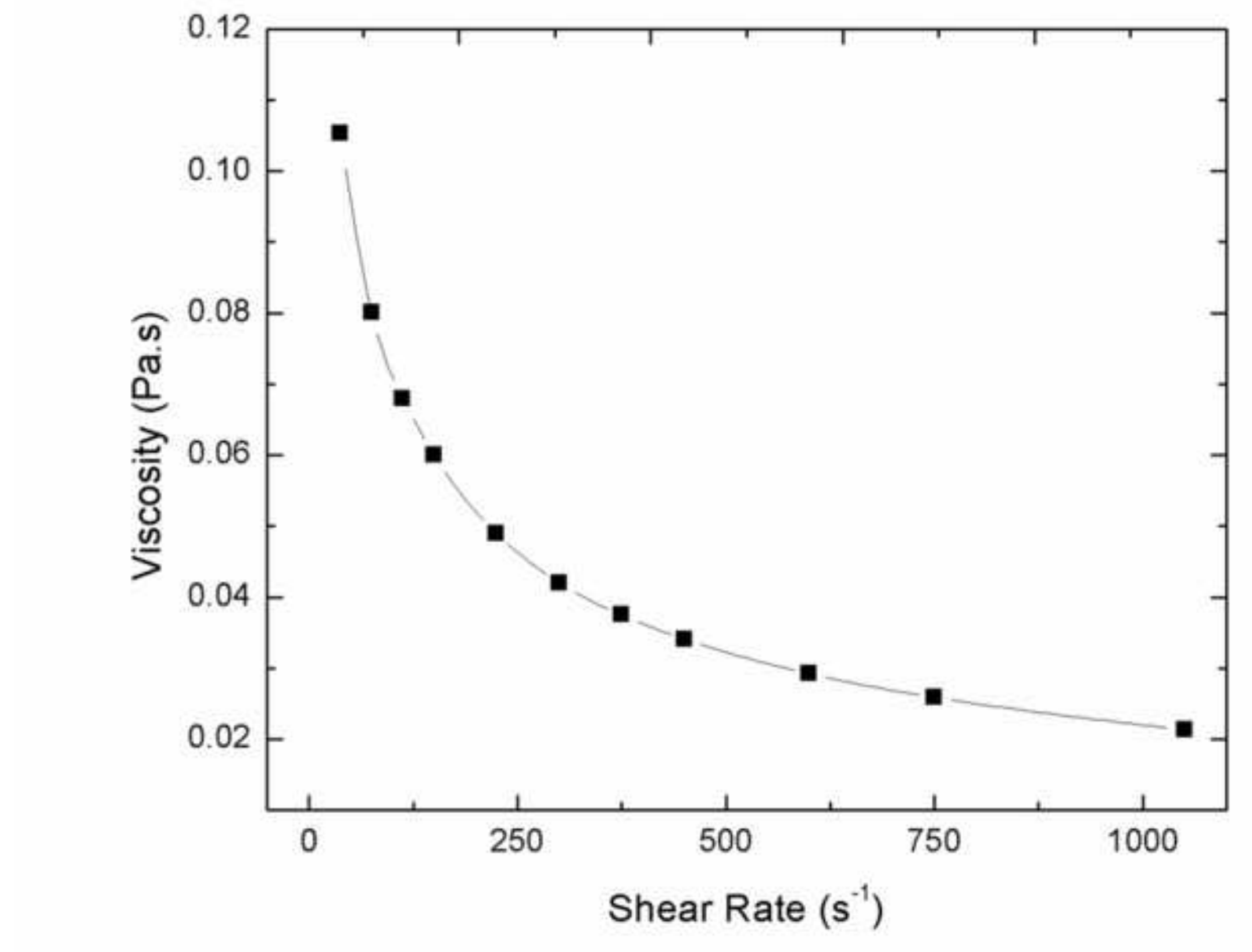




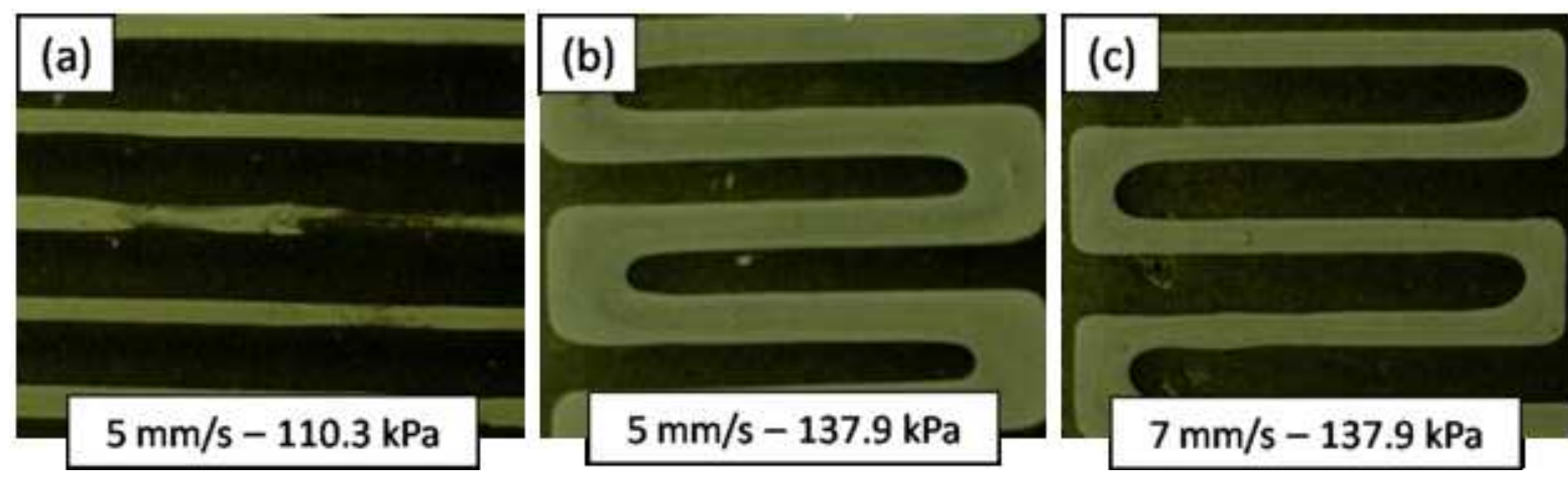

$500 \mu \mathrm{m}$

-

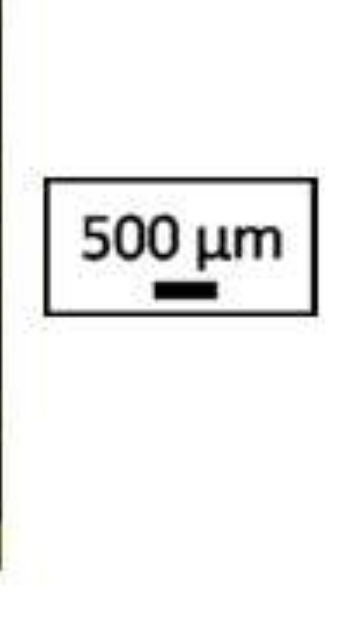




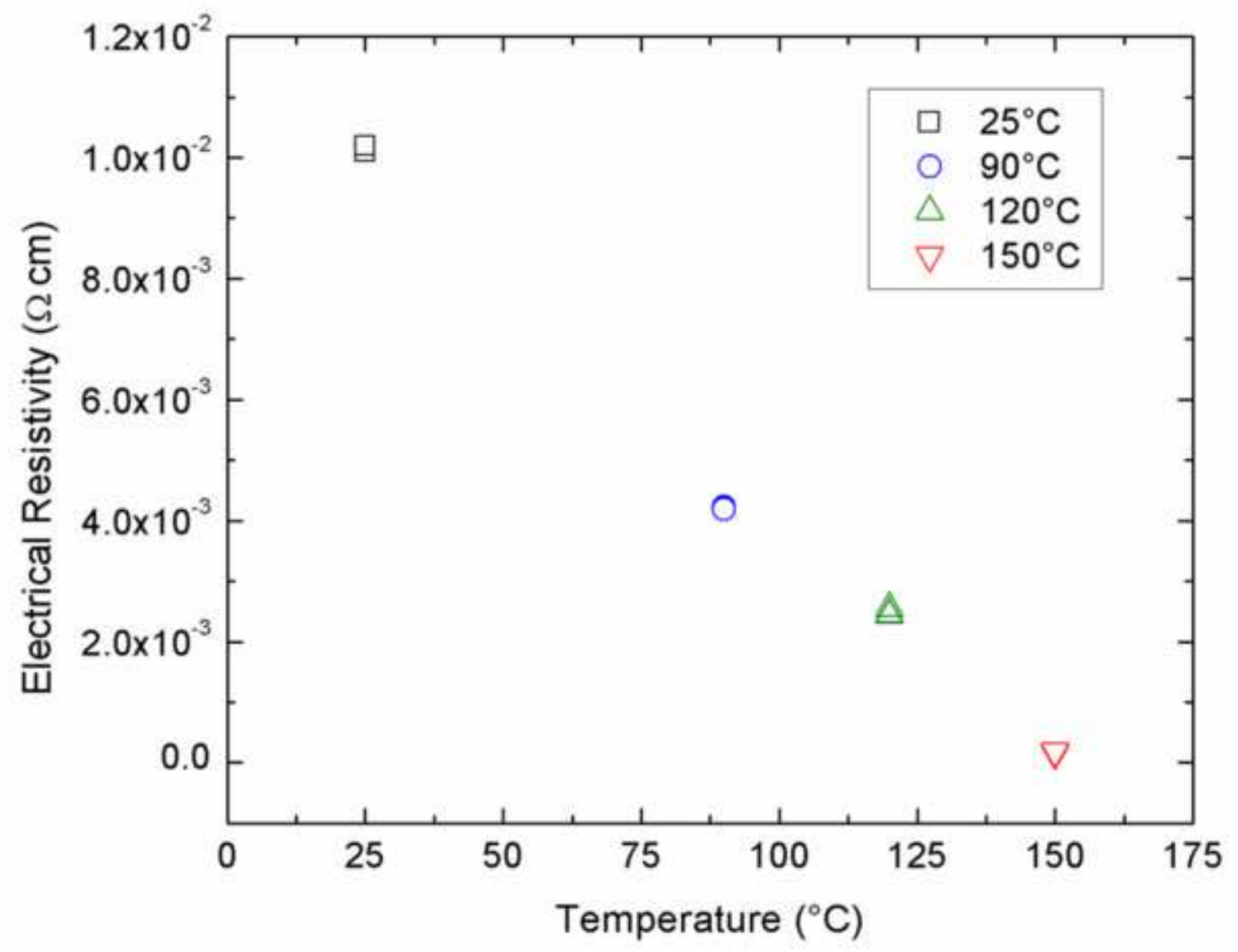



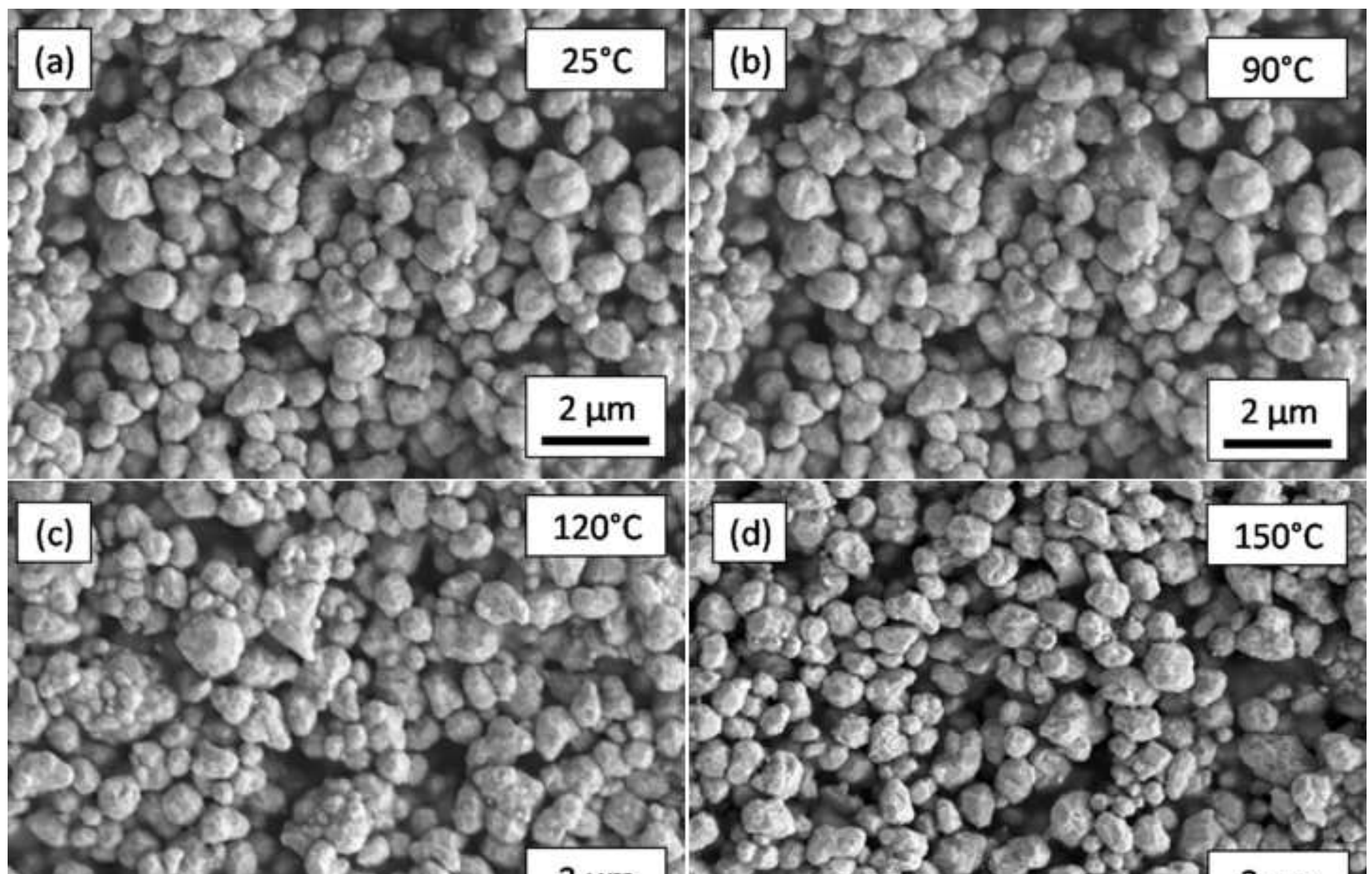

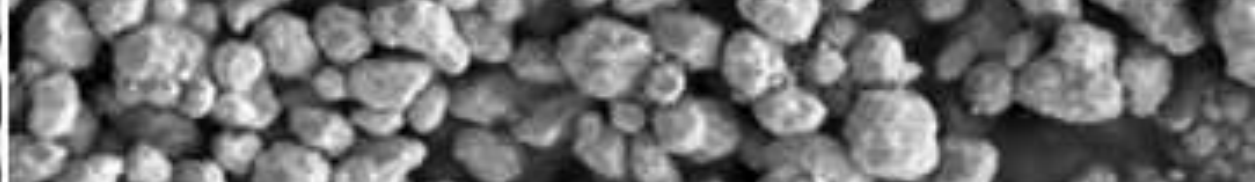
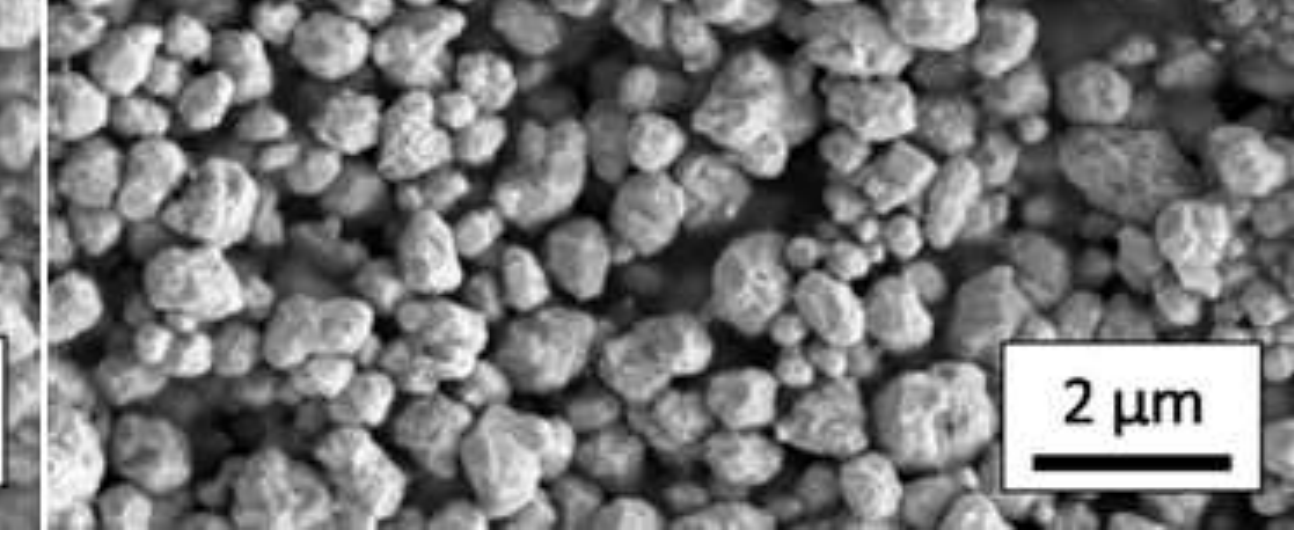

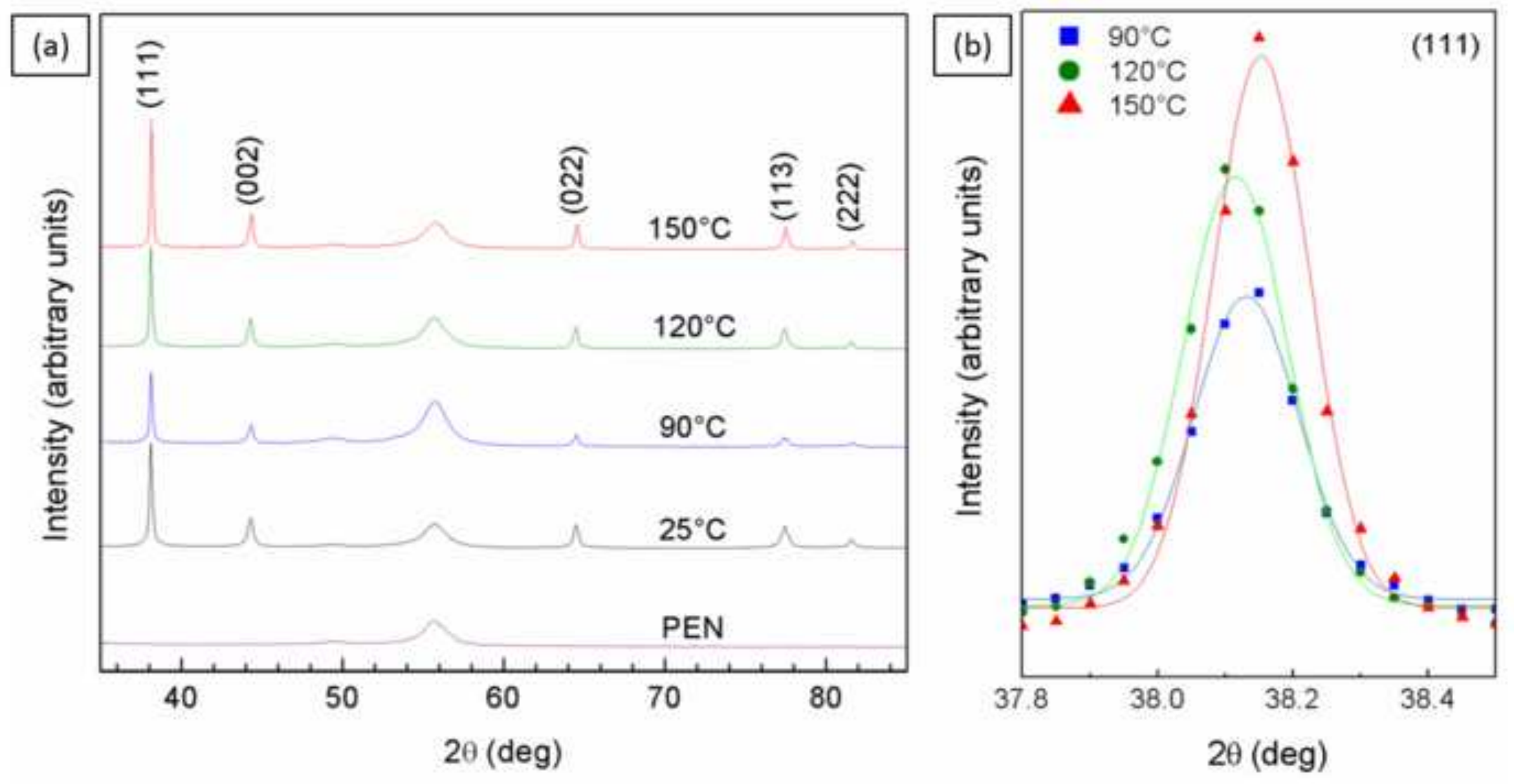


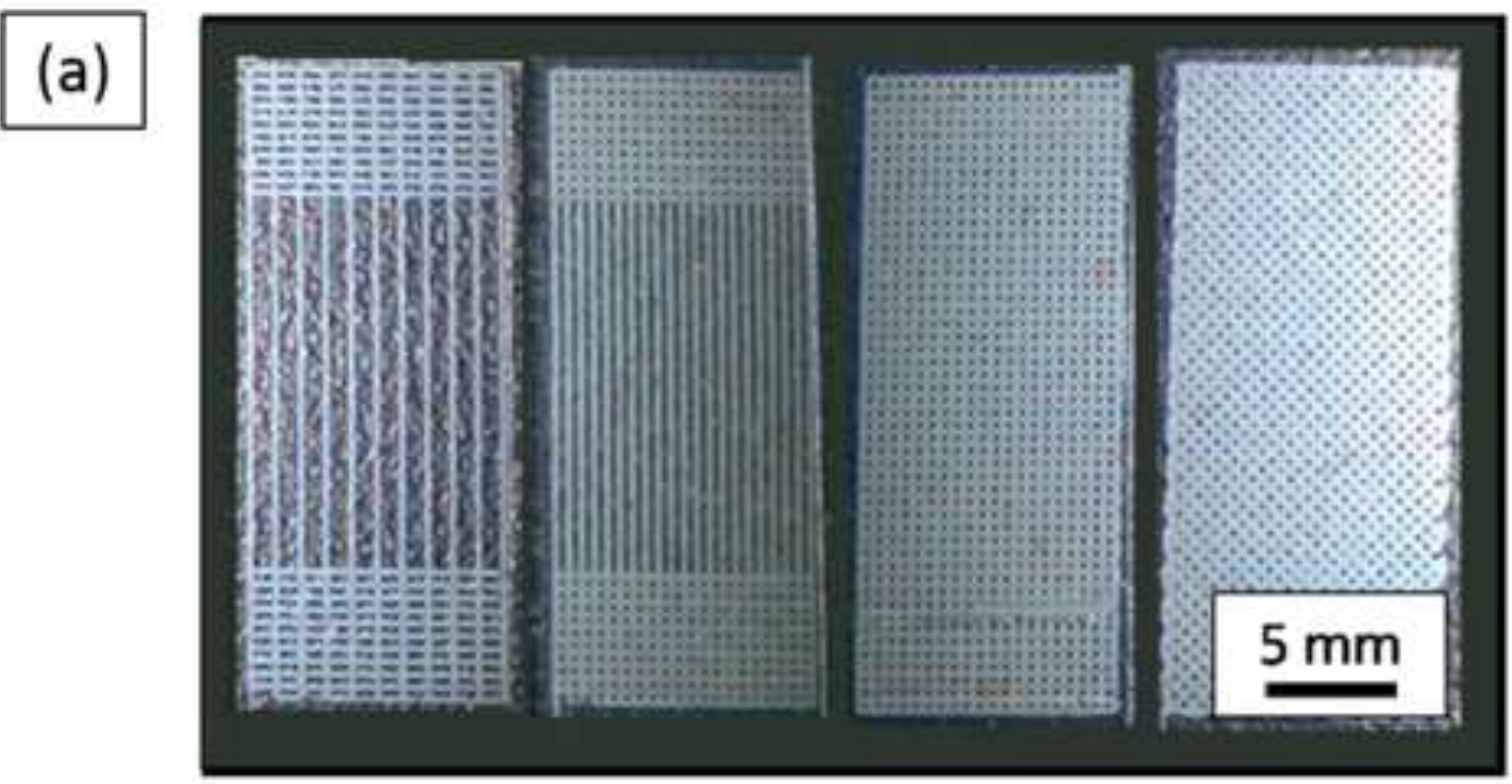

(b)

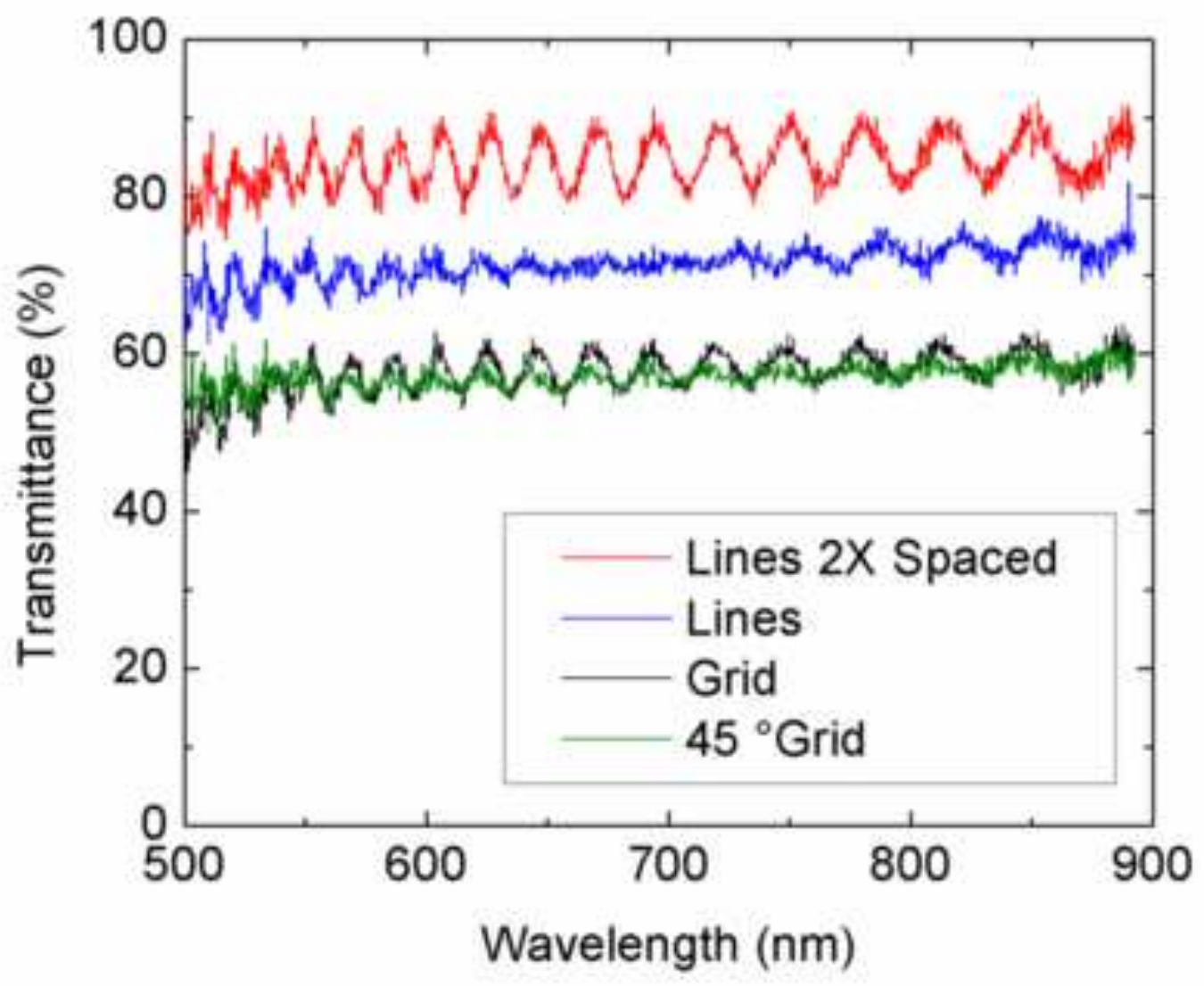



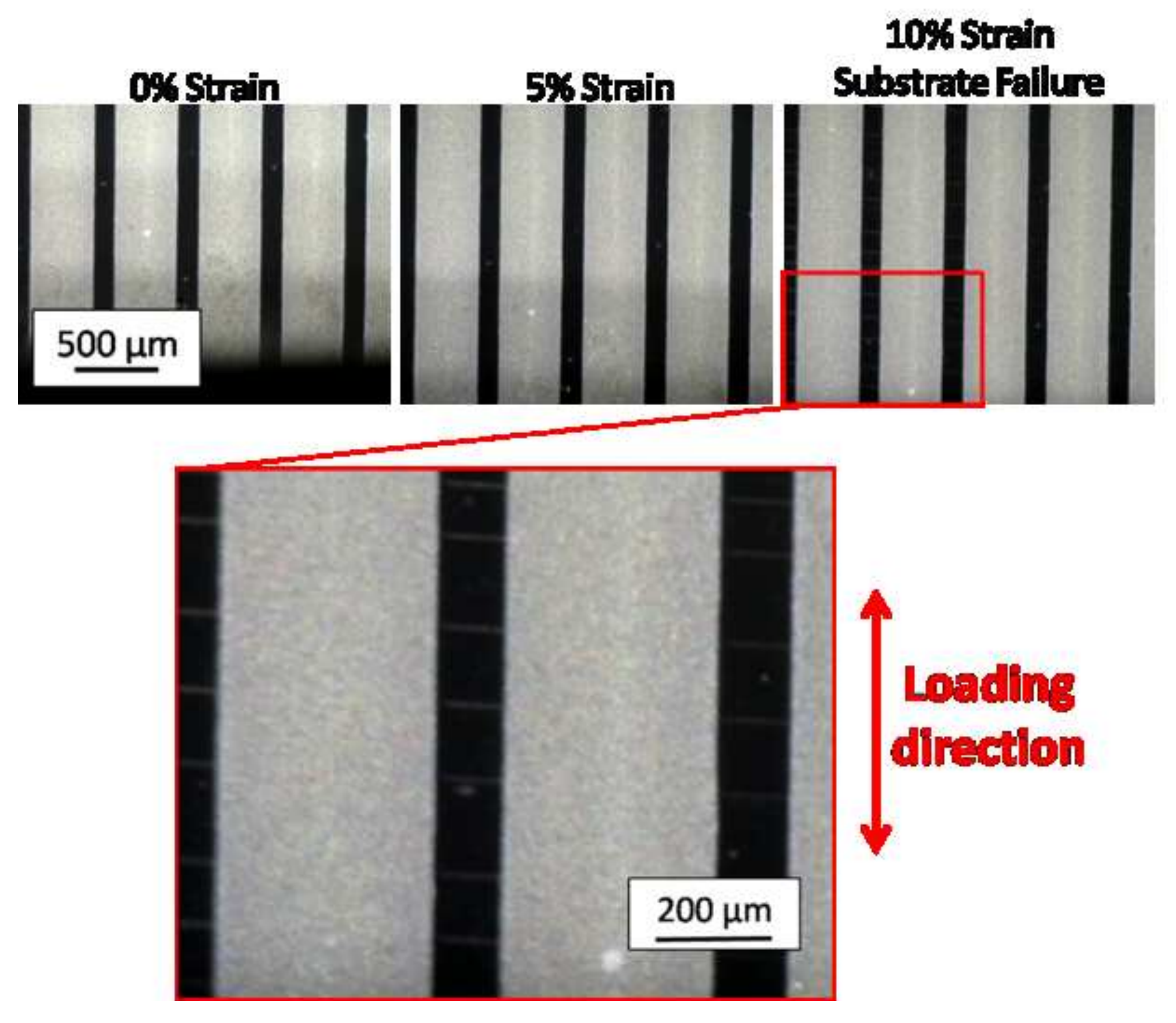

Loading

direction 
(a)

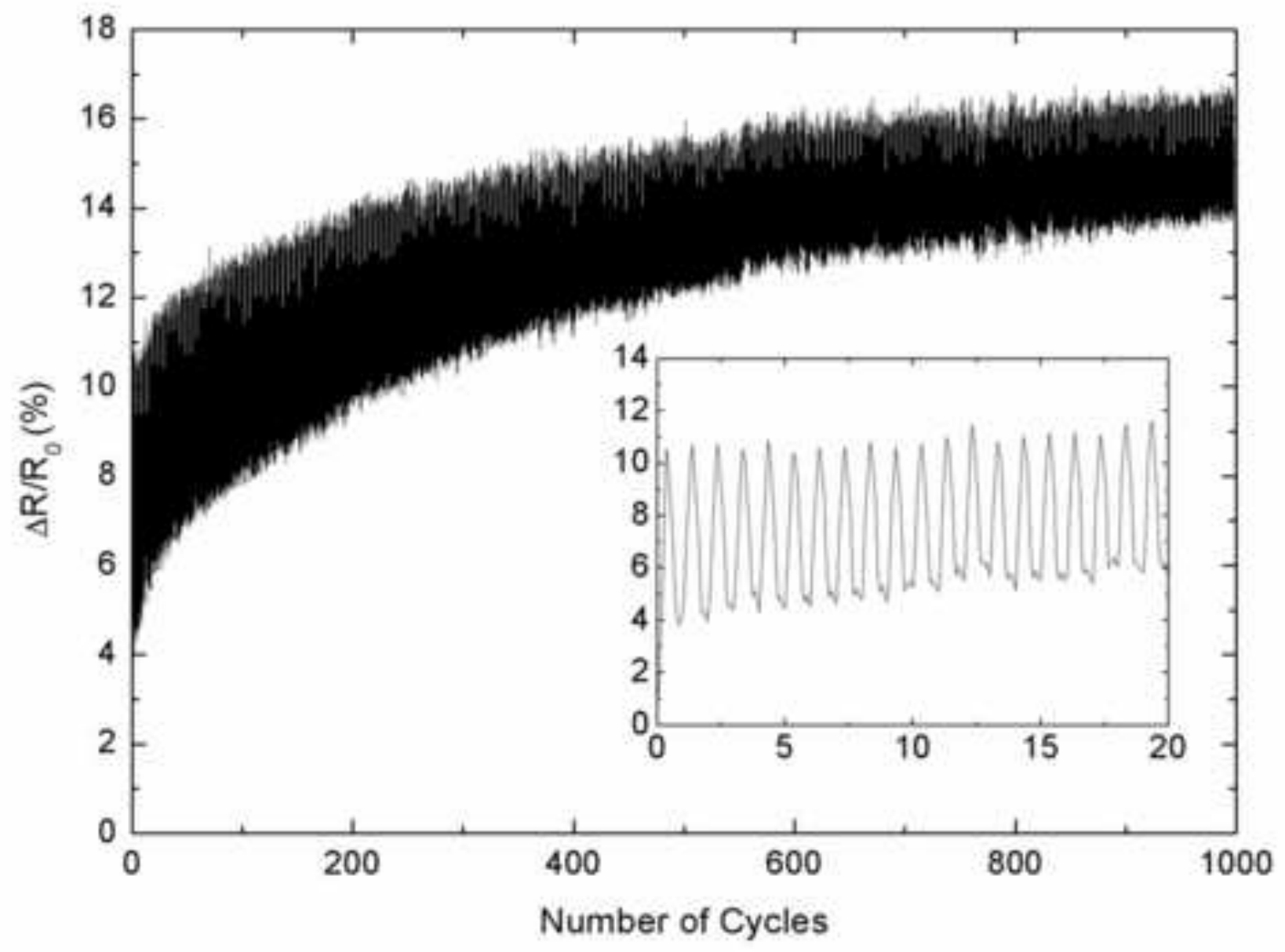

(b)

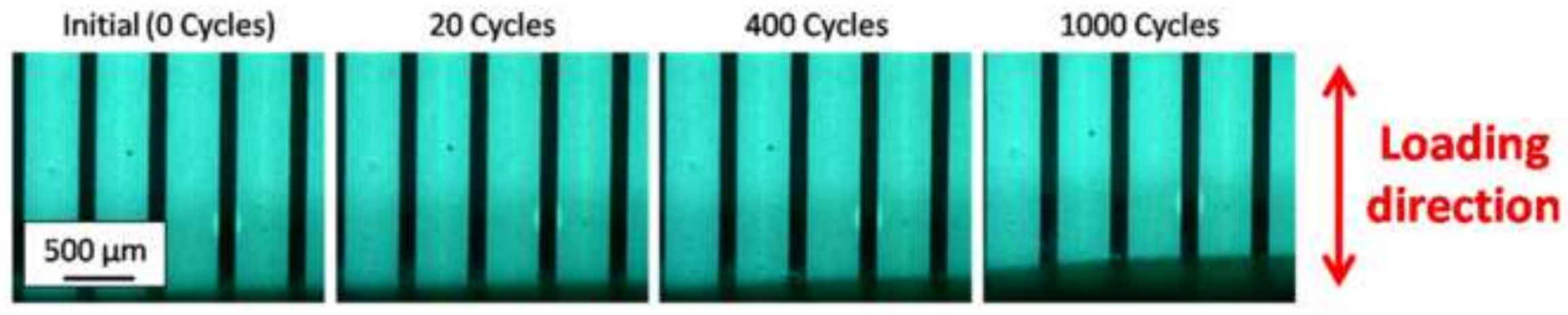



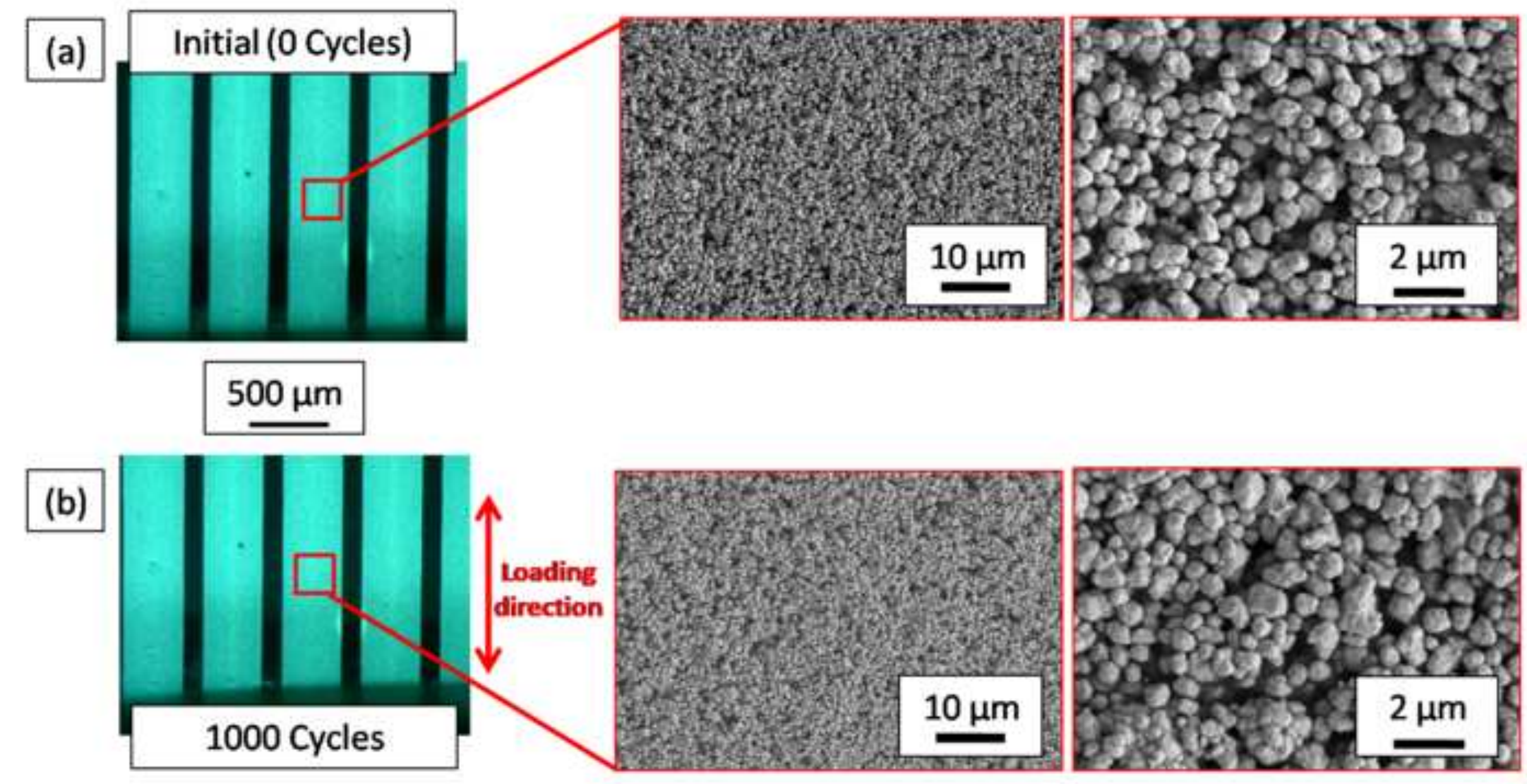